\title{
Critical Current Problems in Neutrons Irradiated HTc Multilayered Superconductors
}

\author{
J. SosnOWSKI* \\ Electrotechnical Institute, M. Pożaryskiego 28, 04-703 Warsaw, Poland
}

\begin{abstract}
In the paper there is given an analysis of the critical current problems in irradiated multilayered high temperature oxide superconductors. Critical current for the direction of current flow inside the layers is investigated basing on analysis of the capturing interaction of pancake vortices with nanodefects, created by fast neutrons irradiation. Analysis of the pinning potential barrier formation is presented. From performed calculations of current-voltage characteristics fitted to measurements the inherent pinning centers concentration and their average dimensions were estimated. The case of perpendicular to layers current has been regarded too, taking into account the intrinsic Josephson junctions formation. The influence of nanodefects concentration on the Josephson penetration depth has been considered, whose length determines the Swihart velocity and current-voltage characteristics of junction.
\end{abstract}

DOI: 10.12693/APhysPolA.130.585

PACS/topics: 74.72.--h, 74.25.Wx, 74.25.Sv

\section{Introduction}

Main efforts in investigations of HTc superconductors are concentrated further on the achieving higher critical temperature and its theoretical explanation. This task results in such recent achievements as new discoveries of superconductors with highest $T_{c}$ reaching $203 \mathrm{~K}$ in $\mathrm{H}_{2} \mathrm{~S}$ [1], surface superconductivity in topological insulators and development of the theoretical approaches based on the Hubbard model and the Green function formalism. However, as it is well known superconducting materials are characterized also by other critical parameters: critical magnetic field and critical current, to theoretical analysis of which much less attention is devoted. While critical magnetic field of the best HTc superconductors is far above availability of the present experimental setups and requirements of superconducting apparatus, critical current plays crucial function from the point of view of applications of HTc superconductors. Previous models of this parameter were based on the Bean critical state approach [2], which can be applied to classical isotropic superconductors. In the present paper there will be given a phenomenological model describing critical current problems in strongly anisotropic multilayered HTc superconductors with nanosized defects. Such nanodefects can be created by the fast neutrons irradiation, whose effect occurs in the accelerators with superconducting windings working in various countries, as LHC in CERN, Nuclotron-NICA in Dubna and especially this problem will concern the huge international project ITER being under construction in Cadarache in France, increasing its actuality. The topic of the influence irradiation on superconducting materials was previously investigated by Weber, see for example [3] and references therein.

*corresponding author; e-mail: j.sosnowski@iel.waw.pl

\section{Current-voltage characteristics of multilayered HTc superconductors with nanodefects}

High temperature copper-oxide superconductors are multilayered materials, in which transport current phenomena are quite different for intralayers processes and for interlayers current flow. Both these effects are influenced by nanodefects created by fast neutrons irradiation, which will be shown in this paper. Intralayers currents are determined by the capturing interaction of pancake type vortices with nanodefects shown in Fig. 1.

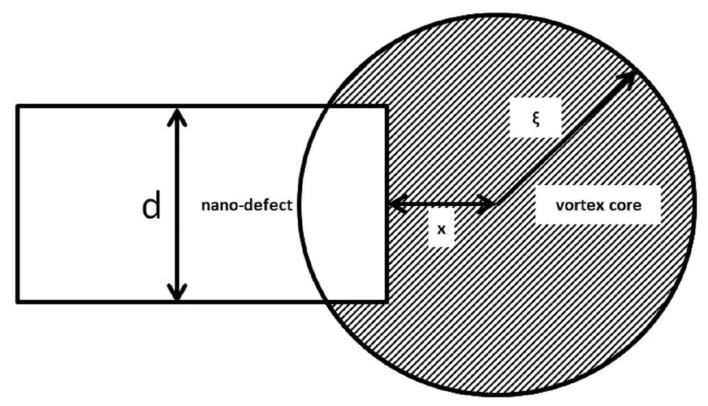

Fig. 1. The geometry of the capturing process of pancake vortex shifted on the length $x$ in respect of initial position.

The deflection $x$ of the pancake vortex core of the radius equal to the coherence length $\xi$ against the capturing this nanodefect center leads to the enhancement [4] of the energy of system $U$, which is described by the following relation:

$$
\begin{aligned}
& U_{1}(x)=\frac{\mu_{0} H_{c}^{2}}{2} l \\
& \quad \times\left[d x-\xi^{2} \arcsin \frac{d}{2 \xi}-\frac{d \xi}{2} \sqrt{1-\left(\frac{d}{2 \xi}\right)^{2}}\right]
\end{aligned}
$$


for the distance $0<x<x_{c}=\xi \sqrt{1-\left(\frac{d}{2 \xi}\right)^{2}}$ and for $x>x_{c}$ :

$$
U_{2}(x)=\frac{\mu_{0} H_{c}^{2}}{2} l \xi\left(x \sqrt{1-\left(\frac{x}{\xi}\right)^{2}}-\arcsin \sqrt{1-\left(\frac{x}{\xi}\right)^{2}}\right)
$$

$H_{c}$ denotes here the thermodynamic critical magnetic field, $l$ is thickness of superconducting layer, $d$ is width of nanodefect. This potential energy describing the vortex capturing causes the appearance of energy barrier $\Delta U$, which should pass vortex during the flux creep process. Energy barrier is expressed by the following equation taking into account the change of the Lorentz force potential during the pancake vortex movement and elasticity energy of vortex lattice connected with its capturing

$$
\begin{gathered}
\Delta U(i)=\alpha_{e} \xi^{2}\left(\sqrt{1-i^{2}}-2\right) \sqrt{1-i^{2}}+\frac{\mu_{0} H_{c}^{2}}{2} l \xi^{2} \\
\times\left\{-\arcsin i+\arcsin \left(\frac{d}{2 \xi}\right)+\frac{d}{2 \xi} \sqrt{1-\left(\frac{d}{2 \xi}\right)^{2}}\right. \\
\left.-i\left[\sqrt{1-i^{2}}+\arcsin \left(\frac{d}{2 \xi}\right)+\frac{d}{2 \xi} \sqrt{1-\left(\frac{d}{2 \xi}\right)^{2}}-\frac{\pi}{2}\right]\right\} .
\end{gathered}
$$

Equation (3) has been written in the current representation, in which $i=j / j_{c}$ is reduced to critical density $j_{c}$, transport current density $j$. Parameter $\alpha_{e}$ describes the elasticity module of the vortex lattice. In derivation of this relation there has been used renormalizing procedure leading to vanishing of the energy barrier for critical current density, similarly as used in collective pinning theory. In Fig. 2 there is shown the dependence of the potential barrier $\Delta U$ on the reduced current density for various values of the elasticity force coefficient $\beta$ in reduced units: $\beta=\frac{2 \alpha_{e}}{\mu_{0} H_{c}^{2 l}}$. The decrease of the potential barrier with rigidity of the elasticity properties of the vortex lattice reflects the fact that elasticity force reduces the capturing effect. Expression (3) describing the potential barrier for flux creep process $\Delta U$ allows then to determine the current-voltage characteristics of multilayered HTc superconductor.

This model has been applied then for the analysis of the current-voltage characteristics of Bi-based HTc superconductors. Calculations have been performed in the function of magnetic field and compared with experimental data measured at liquid nitrogen temperature on $\mathrm{Bi}_{1.6} \mathrm{~Pb}_{0.3} \mathrm{Sr}_{2} \mathrm{Ca}_{2} \mathrm{Cu}_{3.06} \mathrm{O}_{10}$ superconductor. The results shown in Fig. 3 indicate good agreement between model and experiment. In the fitting procedure have been used parameters: average size of the defects acting as the pinning centers and their concentration. For data shown in Fig. 3 the values of these parameters were equal to $d=12 \mathrm{~nm}$ and inherent concentration of defects in the sample $n=3 \times 10^{10} \mathrm{~cm}^{-2}$. The value of width of defect is larger than twice value of coherence length, for

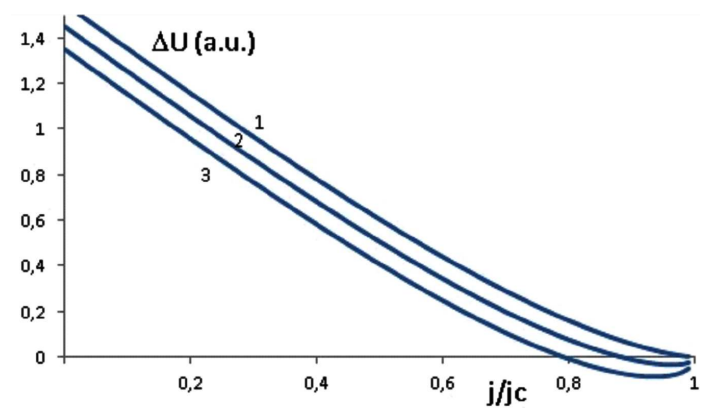

Fig. 2. Influence of reduced current density on energy barrier $\Delta U$ for various values of elasticity force coefficient $\beta$ : (1) $\beta=0$, (2) $\beta=0.1$, (3) $\beta=0.2$.

$\mathrm{BiSrCaCuO}$ compound taken as $\xi=2.57 \mathrm{~nm}$, which allows to neglect in calculations the size dependent corrections in Eq. (3). Also low applied magnetic field permitted to omit the elasticity properties of vortex lattice. In Fig. 4 there are shown from other side the results of calculations of influence size of nanodefects on critical current versus neutrons irradiation concentration. Theoretically predicted initial increase of the critical current with irradiation dose is in qualitative agreement with experimental data given in [5] describing the influence of protons irradiation on $\mathrm{Nb}_{3} \mathrm{Sn}$ wires.

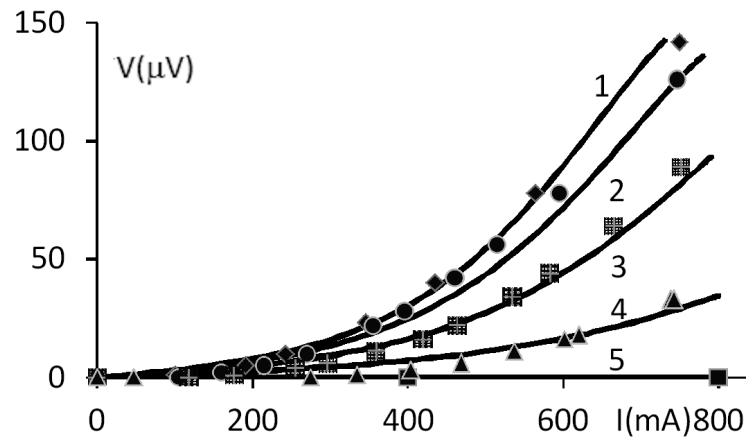

Fig. 3. Comparison of theoretical (line) and experimental (points) current-voltage characteristics for $\mathrm{Bi}_{1.6} \mathrm{~Pb}_{0.3} \mathrm{Sr}_{2} \mathrm{Ca}_{2} \mathrm{Cu}_{3.06} \mathrm{O}_{10}$ superconductor at liquid nitrogen temperature for various magnetic fields: (1) $B=$ 35, (2) $33 \mathrm{mT}$, (3) $24 \mathrm{mT}$, (4) $13.5 \mathrm{mT}$, (5) $0 \mathrm{mT}$.

Flow of the perpendicular to the layers current has been analyzed considering the long intrinsic Josephson junctions formation between the adjacent HTc layers. Phase difference $\Delta \Phi$ of wave function is described then by the following relations, called sine-Gordon modified equation:

$$
\frac{\partial^{2} \Delta \Phi}{\partial t^{2}}-\frac{\partial^{2} \Delta \Phi}{\partial x^{2}}+\alpha \frac{\partial \Delta \Phi}{\partial t}+\sin \Delta \Phi=\frac{\mathrm{J}}{J_{c}},
$$

$\alpha=\gamma \sqrt{\frac{\hbar}{2 e C j_{c}}}$ is loss coefficient describing normal current flow through junction of conductivity $\gamma$ and capacitance $C$. Solution describing moving vortex [6] in the annular Josephson junction then is 


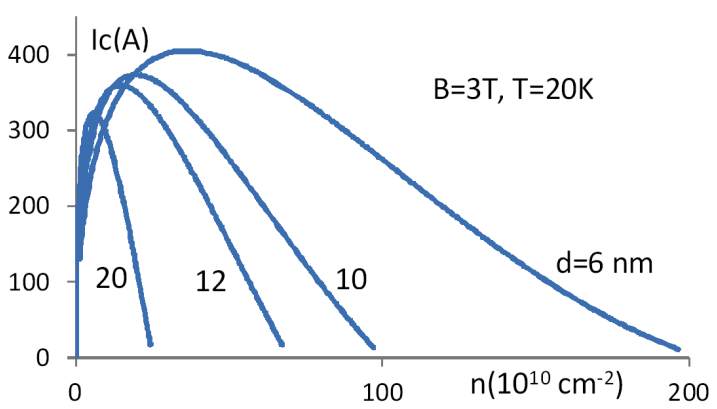

Fig. 4. Theoretical dependence of critical current on irradiation dose versus size of nanodefect.

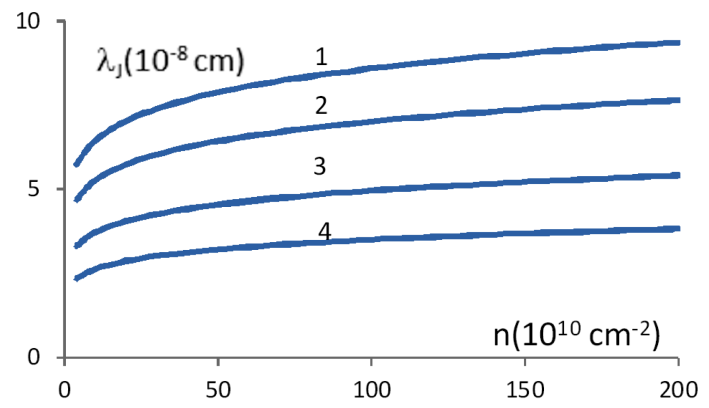

Fig. 5. Calculated dependence of the Josephson penetration depth $\lambda_{\mathrm{J}}$ on irradiation dose versus the London penetration depth in pure limit $\lambda_{\mathrm{L}}$ : (1) $\lambda_{\mathrm{L}}=10^{-5} \mathrm{~cm}$, (2) $\lambda_{\mathrm{L}}=2 \times 10^{-5} \mathrm{~cm},(3) \lambda_{\mathrm{L}}=4 \times 10^{-5} \mathrm{~cm}$, (4) $\lambda_{\mathrm{L}}=$ $6 \times 10^{-5} \mathrm{~cm}$.

$$
\Delta \Phi(x, t)=4 \arctan \left(\frac{x-u t}{\sqrt{1-u^{2}}}\right) .
$$

In Eq. (5) $u$ describes the movement velocity of the Josephson vortex proportional to generated voltage, normalized to the Swihart velocity: $\lambda_{\mathrm{J}} \omega_{p} . \omega_{p}$ is the Josephson plasma frequency, while $\lambda_{\mathrm{J}}$ is the Josephson penetration depth in multilayered HTc superconductor related to the London penetration depth in dirty limit $\lambda_{d}$ according to

$$
\lambda_{\mathrm{J}}=\sqrt{\frac{\Phi_{0}}{2 \pi \mu_{0}\left(d_{i}+2 \lambda_{d} \operatorname{coth} \frac{d_{s}}{\lambda_{d}}\right) j_{c}}} .
$$

$\Phi_{0}$ is flux quantum, $d_{i}$ - thickness of buffer layer, $d_{s}$ - thickness of superconducting layers. $\lambda_{d}$ for dirty superconductors is function of mean free electron path, approximated here as lattice constant $a=1 / \sqrt{n}$ of regularly ordered nanodefects

$$
\lambda_{d}=0.615 \lambda_{\mathrm{L}} \sqrt{\frac{\xi}{a}} .
$$

Results of calculations the influence of neutrons irradiation dose on the Josephson penetration depth $\lambda_{\mathrm{J}}$ in the function of the London penetration depth in pure case $\lambda_{\mathrm{L}}$ is given in Fig. 5. This relation according to previous considerations influences the normalized velocity $u$ and in this way the current-voltage characteristics for perpendicular direction of current to superconducting layers.

\section{Conclusions}

In the paper there have been analyzed the peculiarities of the transport current flow through the multilayered HTc superconductors. Influence on the current-voltage characteristics of the fast neutrons irradiation creating nanosized defects has been regarded. For intralayers currents the interaction of these defects with pancake vortices was analyzed and gave the numerical results in well agreement with experimental data. Perpendicular, interlayers currents were analyzed basing on the Josephson tunneling effects taking into account influence of defects on vortices velocity through the variation then of the Josephson penetration depth.

\section{References}

[1] A.P. Drozdov, M.I. Eremets, I.A. Troyan, V. Ksenofontov, S.I. Shylin, Nat. Lett. 525, 73 (2015).

[2] C.P. Bean, Phys. Rev. Lett. 8, 250 (1962).

[3] D.V. Kulikov, Yu.V. Trushin, F.M. Sauerzopf, M. Zehetmayer, H.W. Weber, Physica C Supercond. 355 , 245 (2001).

[4] J. Sosnowski, Mod. Phys. Lett. B, 2016, submitted.

[5] T. Spina, C. Scheuerlein, D. Richter, L. Bottura, A. Ballarino, R. Flükiger, J. Phys. Conf. Series 507, 022035 (2014).

[6] N.F. Pedersen, IEEE Trans. Magn. 27, 3328 (1991). 